\title{
Reliability Analysis for Systems With Large Hydro Resources in a Deregulated Electric Power Market
}

\author{
Camino González, Jesús Juan, José Mira, Francisco J. Prieto, and María J. Sánchez
}

\begin{abstract}
This work describes a procedure that determines the optimal allocation for the yearly energy resulting from random water inflows to the different subperiods of a year so that the expected benefits are maximized. Its main idea is to distribute the energy stored in reservoirs in each period into two parts: one is directly sold in the energy market, while the other is made available to cover any unplanned outages of thermal units. The method proposed fulfills two objectives, to distribute the hydro energy optimally according to economic criteria and to assess the impact of new market rules on the reliability of an electric system. The procedure will be illustrated by an example based on the Spanish generating system.
\end{abstract}

Index Terms-EENS, LOLE, loss of energy, loss of load, reserves allocation, planning.

\section{INTRODUCTION}

$\mathbf{E}$ LECTRIC systems in many countries have been undergoing radical changes in their market structures and regulatory laws. The new process has replaced traditional expansion planning and operation procedures based on centralized decisions by market oriented approaches [1], [2]. In a common setup, generators bid prices for their energy production, typically on an hourly basis for the next day, in a wholesale energy market. Units are then loaded by increasing price until demand is met. The dispatched generators are remunerated on the basis of the system spot price, which corresponds to the bid of the most expensive loaded unit.

When making decisions with respect to supply it is necessary to distinguish between thermal and hydro units.

The energy amount and price bid by a thermal unit for a given period is essentially a function of its generation costs, modified to take into account constraints such as fuel availability, emission limits, etc... The energy that the thermal unit can supply is mostly limited by its capacity, again with small changes that may need to be introduced to satisfy fuel and emission constraints. In contrast, the peculiar features of hydro units require a significantly different treatment. Firstly, the capacity of the unit is a function of the unit generation capacity, the reservoir storage capacity and water inflows; for long-term studies, this last random factor is the most significant one. Moreover, an important role of these units is to store and transfer energy from

This work was supported by the Project PB-98-0728 of the Ministerio de Educación, Cultura y Deporte, Spain.

C. González, J. Juan, J. Mira, and M. J. Sánchez are with the Escuela Téc. Sup. Ing. Industriales, Universidad Politécnica de Madrid, 28006 Madrid, Spain (e-mail: camino@etsii.upm.es).

F. J. Prieto is with the Department de Estadística y Econometría, Universidad Carlos III, 28903 Getafe, Spain. one period to another. Also, generating costs are negligible. As a consequence, decisions on bids for a given period must be based on alternative uses of the stored water in future periods. Thus, when scheduling the bids of a hydro system there is not alternative to a joint analysis over different periods, even in long-term studies.

In addition to the main market, there exists a secondary one known as reserve market. The reserve market assesses the reliability of the electric energy system, where participants explicitly bid prices at which they are willing to supply capacity reserve. The generators will attempt to exploit any potential profitmaking opportunities through their bidding behavior, with the goal of maximizing their expected profit.

From the point of view of their reliability, hydro and thermal power plants require a different treatment: outages in hydro stations are so unfrequent in comparison with thermal power plants that their outage rate can be treated as zero. Hydro plants are used as regulators of the system in order to meet peak loads and to cover outages of the thermal units.

In hydrothermal systems with an important proportion of hydro energy, such as the Spanish system, many other important aspects of the systems are very sensitive to the distribution of the yearly hydro energy among the different subperiods of the year: for example, the price resulting from the spot market, the maintenance scheduling of the thermal units or the reliability analysis. The purpose of this work is to find an optimal strategy for this allocation, from a economic point of view, which can be a starting point for other analysis that depend on this distribution. Of particular interest, this distribution could be used to assess the impact of the market on the reliability of the system [3], [4].

It is natural and common to use years as the standard period for planning and programming studies. Bearing this in mind and also with the purpose of simplifying the presentation of the paper, we shall use years as the reference period for our study. The year is divided into subperiods of equal length (weeks, fortnights, or months).

The models described in this paper have been developed as tools for the study of long-term decisions in energy generation systems such as those related to investment, capacity expansion, reliability studies, etc. Our emphasis is in the determination of an overall policy for the use of the hydro energy available during the period under study. As a consequence, several simplifications in the modeling of the system have been adopted to take into account the limitations in the availability of information for long-term studies and to reduce the size and complexity of the resulting problem. 
Regarding the hydro system, we assume the following.

1) All the hydro plants are modeled as one single unit which is featured by an equivalent capacity and a certain amount of available energy [3], [5], [6]

2) For different hydro energies in a subperiod the capacity is constant.

This approach provides significant computational efficiency gains while still allowing the consideration of the energy limitations inherent to the hydro units. Some constraints can be included to ensure feasible solutions, for example, minimum and maximum values for the amount of energy allotted to each subperiod or some bounds for the hydro capacity for the subperiods.

With respect to the markets, we have considered that the behavior of the different individual competitors is not very relevant; it is assumed that all of them will behave in a rational manner within a perfect competition framework. We also assume that prices for energy in the reserve market are higher than those in the main energy market within each period. As a consequence, the best policy is to sell through the reserve market all the hydro energy that this market may require, and the remaining available hydro energy is sold to the energy market. The amount of energy demanded by the reserve market has been modeled as the consequence of the (un)availability of the thermal units, as we discuss below. To represent the energy markets in a compact manner, we have chosen to use the (monthly) load duration curves for the demand. Regarding prices, we make the assumption that the bidding prices for the thermal units are constant within each period. Under this assumption, there is a direct correspondence between demand and prices, and in particular any ordering of the prices is associated with an equivalent ordering of the demand. As a consequence, the load duration curve corresponds to an equivalent "price duration curve" and for example a shaving of one of these curves induces an equivalent shaving in the other curve finally, we will assume that the unavailability of a given thermal unit will always be covered through energy generated from the reserve market.

In this paper, we compare two strategies for the allocation of the hydro energy: first, we apply the procedure explained in [4], by which the reliability indices are optimized and maintained constant throughout the year. In the second procedure, we seek optimization from the economic point of view. Both procedures are applied to the spanish market, in which three possible hydro scenarios (wet, normal, dry) are considered.

This paper is structured as follows; in Section II, a new hydro allocation strategy is described, in Section III we present the application and comparison exercise for the Spanish case and Section IV are the conclusions.

\section{Two Hydro Allocation StrategiES}

\section{A. Introduction}

In both cases, the problem can be stated as the determination of the optimal hydro energy production for a subperiod (week, fortnight, month ... .) over a one year time span so that the annual hydraulic constraints are satisfied. The analysis is carried out for a one year length future period. Let $W$ denote the available hydro energy forecast for the year of interest. For simplicity, $W$ will be considered initially as a fixed and known value. Let the

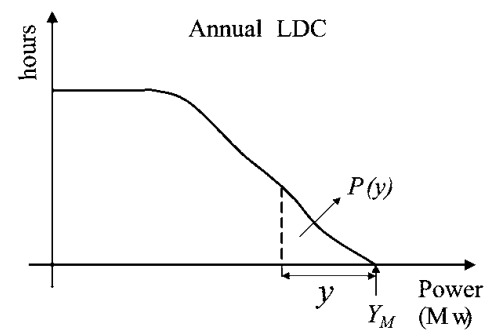

Fig. 1. Peak shaving method. The discount $y$ is obtained from the annual peak load $Y_{M}$.

year be divided into $m$ subperiods of equal duration. The objective is to determine the values $W_{k}, k=1, \ldots, m$, of hydro energy allotted to each subperiod $i$, while satisfying the constraint

$$
\sum_{k=1}^{m} W_{k}=W
$$

Each subperiod is described by the load curve, the thermal system composition and the available hydro capacity which are supposed to be known and fixed.

Hydro plants are used to satisfy specific loads and to cover outages of thermal units. From and economic point of view the behavior of these hydro units is optimized if the generation is conducted in those hours when the price is largest, or alternatively when the demand is largest.

We shall decompose the hydro energy of each subperiod $W_{k}=P_{k}+O_{k}$, where $P_{k}$ is the energy bidded to cover the load peaks and $O_{k}$ the one assigned to cover the failures of the thermal units.

The load model used most extensively is the Load Duration Curve (LDC), $L_{k}$ [5], where $t=L_{k}(x)$ measures the time during which demand exceeds a particular level of load $x$ for the subperiod $k$. Load curves are determined from historical data which are usually obtained on a routine basis at hourly intervals by the electric utilities.

The area under the load curve $L_{k}$ for the interval $\left[x_{1}, x_{2}\right]$

$$
s=A\left(L_{k}, x_{1}, x_{2}\right)=\int_{x_{1}}^{x_{2}} L_{k}(x) d x
$$

gives the demanded energy $s$ corresponding to this interval.

A different thermal system is asumed for each period due to maintenance. The optimal allocation in the different subperiods is equivalent to peak-shaving for the annual load curve with discount $y$. See Fig. 1. This implies bidding an amount of energy $P(y)$ during the hours with demand larger than $Y_{M}-y$. The area under the $L D C$ between $Y_{M}-y$ and $Y_{M}, P(y)$, represents the energy supplied with hydro resources to cover load peaks.

From $P(y)$ and from the corresponding load curve $L_{k}$ of the subperiod $k$, we can obtain the $P_{k}(y)$ values, so that $P(y)=$ $\sum_{k=1}^{m} P_{k}(y)$.

The rest of the hydro energy will be used to cover the failures of the thermal units. The thermal power an electric system is able to supply during a subperiod is a random variable which depends on the availability of the plants in the system. For simplicity, only two states are assumed for each thermal unit: oper- 
ating or failed. If the forced outage rate of unit $i$ is $p_{i}$, and $c_{i}$ its nominal capacity, the power failed or not supplied by this unit is a random variable $U_{i}$ with the following probability distribution:

$$
f_{i}(u)= \begin{cases}1-p_{i}, & u=0 \\ p_{i}, & u=c_{i} .\end{cases}
$$

If the system has $n$ units, the random variable thermal power not supplied by the system, $U$, is

$$
U=U_{1}+U_{2}+\cdots+U_{n} .
$$

In the case of independent $U_{i}$ variables, the probability distribution of $U$ will be given by the convolution of functions $f_{i}(2)$, [8], [9]. If the capacities of the thermal units are $c_{1}, c_{2}, \ldots, c_{n}$, respectively, we define $c_{T}=c_{1}+c_{2}+\cdots+c_{n}$ as the total nominal capacity, and $S=c_{T}-U$ as the supplied or nonfailed generating capacity random variable.

\section{B. Hydro Distribution Under Economic Optimality}

The thermal system should cover energy demand once the amount assigned to the hydro system through peak-shaving has been discounted. The modified load curve $M_{k}$ for period $k$ is defined as follows through the load curve $L_{k}$ of the period

$$
M_{k}(x)= \begin{cases}L_{k}(x), & x<Y_{M}-y \\ 0, & x \geq Y_{M}-y\end{cases}
$$

where $y \leq c_{H}$, being $c_{H}$ the hydro capacity. Thus, if the random variable $\bar{S}$ takes the value $s$, that is the power supplied by the thermal system is $s$, less than $Y_{M}-y$, the area under the modified curve $M_{k}$ between $s$ and $Y_{M}-y$ corresponds to the energy demanded and not served

$$
E_{k}(s)=\int_{s}^{Y_{M}-y} M_{k}(u) d u
$$

Due to the random character of supply, $E_{k}$ (energy not supplied by the thermal system) is a random variable and its expectation is

$$
\sum_{j} E_{k}\left(c_{T}-x_{j}\right) \operatorname{Pr}\left\{U=x_{j}\right\} .
$$

Given the thermal system and the load duration curve of the subperiod $k$ the above value only depends on the discount $y$, and we use this amount (4) as the hydro energy used to cover thermal outages

$$
O_{k}(y)=\sum_{j}\left(\int_{c_{T}-x_{j}}^{Y_{M}-y} M_{k}(u) d u\right) \operatorname{Pr}\left\{U=x_{j}\right\} .
$$

The function $O_{k}$ is a nonincreasing function, $O_{k}(0)$ is the expected value of the energy not supplied by the thermal system due to failures when the discount of energy demand is zero $(y=0)$. If we call $O(y)=\sum_{k=1}^{m} O_{k}(y)$, when $O(0)>W$, the system does not have enough capacity to cover demand even if we use all the hydro energy to cover the failures of the thermal units. On the other hand, and this is now the situation we focus on, when $O(0)<W$, this amount is enough to cover the thermal failures and also to use part of its capacity to cover the load

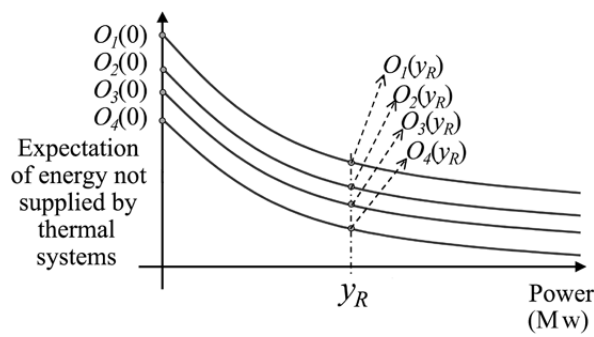

Fig. 2. Expected value of energy not supplied by the thermal system as a function of $y$, the peak-shaving discount.

curve. As $P(y)=\sum_{k=1}^{m} P_{k}(y)$, assuming that $P(y)$ is a continuous function of $y$ and $W<P\left(Y_{M}\right)$, that is, the hydro energy available through the year is smaller than the total energy demanded, and noting that $P(0)+O(0)-W<0$ and $P\left(Y_{M}\right)+O\left(Y_{M}\right)-W>0$, by continuity there exists a value $y_{R}$, such that

$$
P\left(y_{R}\right)=W-O\left(y_{R}\right) .
$$

This value $y_{R}$ is optimal from the reliability point of view since $W-O\left(y_{R}\right) \geq 0$ and also efficient economically. This value can be obtained iteratively, see Fig. 2 where four periods have been considered. The algorithm is as follows.

1) For each power discount $y$ (starting from the solution $y=0)$ and from each period load curve $L_{k}$, the functions $P_{k}(y)$ and $O_{k}(y)$ are obtained.

2) The function $O(y)+P(y)$ is evaluated; it represents the total hydro energy to be used when the discount $y$ is shaved, and when an optimal allocation of the energy sold to the market $P(y)$ is carried out. As $W$ denotes the annual available hydro energy forecast, the optimal solution will be obtained by ensuring that $O(y)+P(y)$ is as large as possible, that is $O(y)+P(y)=W$. The value of the power discount satisfying the preceding condition is denoted as $y_{R}$.

3) Once $y_{R}$ is known, the values $P_{k}\left(y_{R}\right), O_{k}\left(y_{R}\right)$, and $W_{k}\left(y_{R}\right)=P_{k}\left(y_{R}\right)+O_{k}\left(y_{R}\right)$, will provide for each subperiod the hydro energy bidded to cover load peaks, the hydro energy assigned to cover thermal failures and the total hydro energy respectively.

If the prices per Mw for each period and for each of the components of the hydro energy are known in each period (or can be estimated), one can obtain the value of $y$ which provides the optimal allocation from an economical point of view.

The distribution of hydro energy according to the model just described is established as a function of the variable $y$, it is thus interesting to study how the reliability indicators of the system change with $y$ [3]-[7]. Moreover, one can also study how the new decision rules are influential on reliability.

\section{ReSUlts OF the APPLICATION to the SPANiSh CASE}

The proposed procedure has been applied to analyze the reliability of the Spanish system in the next five years. In this section we show the results for the year 2007. The load curve are obtained from the 2002 data whith an annual increase of $5 \%$ per year. This is approximately the mean value observed in the last 
TABLE I

Monthly Optimal Allocation of Hydroenergy (GWh). Wet YeaR

\begin{tabular}{c||c|c|c|c}
\hline Month & $\mathrm{W}_{k}$ & $\mathrm{O}_{k}$ & $\mathrm{P}_{k}$ & $\mathrm{~W}_{R}$ \\
\hline 1 & 5592.1 & 1482.1 & 4110.0 & 5009.6 \\
\hline 2 & 3554.9 & 1191.2 & 2363.7 & 3764.2 \\
\hline 3 & 3736.8 & 1362.5 & 2374.3 & 4068.8 \\
\hline 4 & 1925.6 & 908.8 & 1016.8 & 2013.3 \\
\hline 5 & 2874.4 & 1097.8 & 1776.6 & 3071.1 \\
\hline 6 & 4030.8 & 1308.4 & 2722.4 & 4071.1 \\
\hline 7 & 4317.6 & 1357.6 & 2960.0 & 3948.0 \\
\hline 8 & 3532.3 & 1253.8 & 2278.5 & 3093.8 \\
\hline 9 & 3167.7 & 1185.1 & 1982.6 & 3496.7 \\
\hline 10 & 3307.1 & 1240.9 & 2066.2 & 3492.8 \\
\hline 11 & 4301.9 & 1274.0 & 3027.9 & 4797.4 \\
\hline 12 & 5741.0 & 1519.2 & 4221.8 & 5255.2 \\
\hline
\end{tabular}

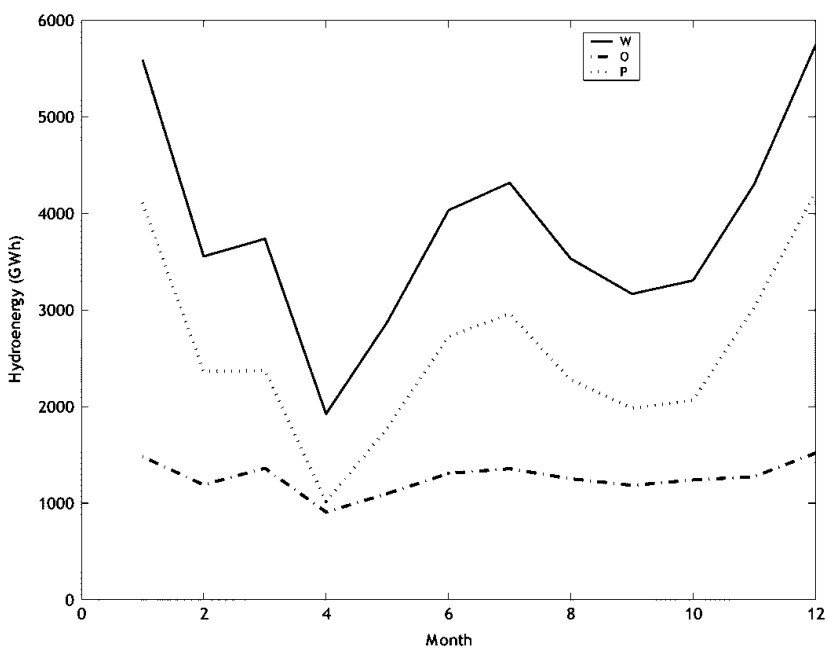

Fig. 3. Monthly optimal allocation of hydroenergy. Wet year.

five years. The thermal system of the analysis is the 2002, in order to study how, if no new thermal units are incorporated to the system, 2007 demand could be met. A brief description of the spanish thermal sytem can be found in [4]. From the point of view of the yearly available hydroenergy, three scenarios have been considered: wet year (46 $082 \mathrm{GWh})$, normal year (31 224 $\mathrm{GWh})$ and dry year (18 $889 \mathrm{GWh})$. These values have been estimated from historical data and assuming that the water inflows follow a lognormal distribution. No short-term significant changes are foreseen for the hydro system.

The reliability index used in the study has been the Loss of Load Expectation (LOLE), i.e., the expected time (in hours) during which the demand of power is greater than the available from the system.

The first step in the analysis is to assign the annual ammount of hydro energy $(W)$ into the different subperiods (months) of the year $\left(W_{k}\right)$. These values are computed following the procedure described in the previous sections, taking into account the fraction of the energy used in peak shaving $\left(P_{k}\right)$ and the one corresponding to the coverage of the failures of thermal units $\left(O_{k}\right)$. Table I and Fig. 3 represent the results of the proposed procedure for the wet year. It can be observed that the values assigned to cover load peaks (ranging from $1016.8 \mathrm{GWh}$ to $4221.8 \mathrm{GWh}$ )
TABLE II

MONTHLY LOLE INDEX IN HOURS. WET YEAR

\begin{tabular}{c||c|c}
\hline Month & Economic & Reliability \\
\hline 1 & 0.00 & 0.01 \\
\hline 2 & 0.07 & 0.01 \\
\hline 3 & 0.17 & 0.01 \\
\hline 4 & 0.00 & 0.01 \\
\hline 5 & 0.02 & 0.01 \\
\hline 6 & 0.03 & 0.01 \\
\hline 7 & 0.00 & 0.01 \\
\hline 8 & 0.00 & 0.01 \\
\hline 9 & 0.08 & 0.01 \\
\hline 10 & 0.03 & 0.01 \\
\hline 11 & 0.08 & 0.01 \\
\hline 12 & 0.01 & 0.01 \\
\hline & &
\end{tabular}

TABLE III

Monthly Optimal Allocation of Hydroenergy (GWh). NoRmal Year

\begin{tabular}{c||c|c|c||r}
\hline Month & $\mathrm{W}_{k}$ & $\mathrm{O}_{k}$ & $\mathrm{P}_{k}$ & $\mathrm{~W}_{R}$ \\
\hline 1 & 4100.9 & 1548 & 2552.9 & 3474.9 \\
\hline 2 & 2395.8 & 1113.4 & 1282.4 & 2611.2 \\
\hline 3 & 2401.6 & 1280.9 & 1120.7 & 2793.5 \\
\hline 4 & 1068.2 & 756.8 & 311.4 & 1203.3 \\
\hline 5 & 1808.7 & 1024.4 & 784.3 & 2015.6 \\
\hline 6 & 2755.6 & 1204.6 & 1551.0 & 2764.5 \\
\hline 7 & 2989.7 & 1262.1 & 1727.6 & 2662.1 \\
\hline 8 & 2313.2 & 1141.6 & 1171.6 & 2006.6 \\
\hline 9 & 2027.2 & 1043.9 & 983.3 & 2377.9 \\
\hline 10 & 2109.7 & 1101.8 & 1007.9 & 2314.9 \\
\hline 11 & 3040.4 & 1268.5 & 1771.9 & 3338.9 \\
\hline 12 & 4212.7 & 1563.7 & 2649.0 & 3660.6 \\
\hline
\end{tabular}

are higher that those assigned to cover thermal outages. This is clearly shown in Fig. 3.

The last column of Table I $\left(W_{R}\right)$ represents the optimal monthly allocation of the yearly hydroenergy from a reliability point of view. These values have been obtained applying the procedure described in [4], using the criterion of leveling the LOLE index in all subperiods. The hydroenergy in this case is reserved just to cover the outage of thermal units. In the following we will call this method reliability criterion and use economic criterium for the one described in this paper. The reliability indices LOLE have been obtained for both cases and are shown in Table II. The results are quite similar. The main conclusion is that the monthly allocation of the hydroenergy $\left(W_{k}\right)$ obtained with the economic criterium is also very acceptable from a reliability point of view. This result is coherent with the considerations taking into account in the resolution of the proposed procedure and detailed in Section II of this work.

Similar analysis have been performed for the two other scenarios: normal (31 $224 \mathrm{GWh}$ ) and dry (18 $889 \mathrm{GWh})$. They are detailed in Tables III, IV and Fig. 4. For normal year, and in Tables V, VI and Fig. 5 for dry years.

For a year of average hydraulicity the differences are significant. The hydro distribution obtained following economic criteria is problematic when covering energy demand, the LOLE index predicts an average of $21.1 \mathrm{~h}$ of failure of supply.

Evidently the problem increases for a dry year. In this case one can observe that the system does not have enough capacity whatever the distribution criterion applied, though with the economic criterion the problem is more acute. This reveals that in 
TABLE IV

MONTHLY LOLE INDEX IN HOURS. NORMAL YEAR

\begin{tabular}{c||c|c}
\hline Month & Economic & Reliability \\
\hline 1 & 0.88 & 0.62 \\
\hline 2 & 2.10 & 0.62 \\
\hline 3 & 5.30 & 0.62 \\
\hline 4 & 0.05 & 0.62 \\
\hline 5 & 0.63 & 0.62 \\
\hline 6 & 0.94 & 0.62 \\
\hline 7 & 0.35 & 0.62 \\
\hline 8 & 0.14 & 0.62 \\
\hline 9 & 1.60 & 0.62 \\
\hline 10 & 0.96 & 0.62 \\
\hline 11 & 6.60 & 0.62 \\
\hline 12 & 1.60 & 0.62 \\
\hline
\end{tabular}

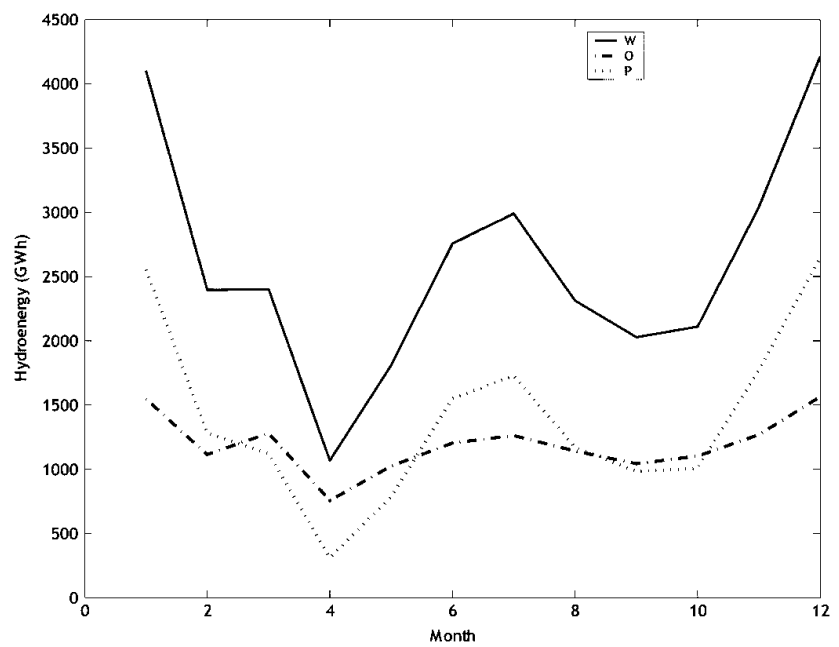

Fig. 4. Monthly optimal allocation of hydroenergy. Normal year.

TABLE V

MONTHLy Optimal Allocation OF HydROENERGY (GWh). DRY YeAR

\begin{tabular}{c||c|c|c||c}
\hline Month & $\mathrm{W}_{k}$ & $\mathrm{O}_{k}$ & $\mathrm{P}_{k}$ & $\mathrm{~W}_{R}$ \\
\hline 1 & 2774.7 & 1420.6 & 1354.1 & 2200.8 \\
\hline 2 & 1425.6 & 1008.6 & 417.0 & 1654.0 \\
\hline 3 & 1300.1 & 1007.0 & 293.1 & $\mathbf{1 7 3 4 . 7}$ \\
\hline 4 & 420.7 & 394.4 & 26.3 & 530.8 \\
\hline 5 & 928.0 & 755.4 & 172.6 & 1139.3 \\
\hline 6 & 1713.4 & 1046.7 & 666.7 & 1679.8 \\
\hline 7 & 1898.4 & 1113.8 & 784.6 & 1594.5 \\
\hline 8 & 1327.1 & 951.2 & 375.9 & 1103.9 \\
\hline 9 & 1123.0 & 848.2 & 274.8 & 1449.1 \\
\hline 10 & 1153.7 & 805.3 & 348.4 & 1337.1 \\
\hline 11 & 1948.6 & 1117.3 & 831.3 & 2128.1 \\
\hline 12 & 2875.6 & 1370.6 & 1505.0 & 2336.8 \\
\hline
\end{tabular}

order to meet demand in 2007, more thermals units should be added to the system.

\section{CONCLUSION}

This paper provides a method to solve the problem of finding the optimal allocation of hydro energy production in every subperiod of a one year time-horizon, according to economic and system reliability criteria. The models are intended to be used as tools for the study of long-term decisions. The proposed method
TABLE VI

MONTHLY LOLE INDEX IN HOURS. DRY YEAR

\begin{tabular}{c||c|c}
\hline Month & Economic & Reliability \\
\hline 1 & 23.4 & 16.21 \\
\hline 2 & 32.4 & 16.21 \\
\hline 3 & 46.8 & 16.21 \\
\hline 4 & 0.2 & 16.21 \\
\hline 5 & 7.8 & 16.21 \\
\hline 6 & 18.4 & 16.21 \\
\hline 7 & 10.6 & 16.21 \\
\hline 8 & 3.3 & 16.21 \\
\hline 9 & 19.7 & 16.21 \\
\hline 10 & 14.0 & 16.21 \\
\hline 11 & 71.8 & 16.21 \\
\hline 12 & 29.3 & 16.21 \\
\hline & &
\end{tabular}

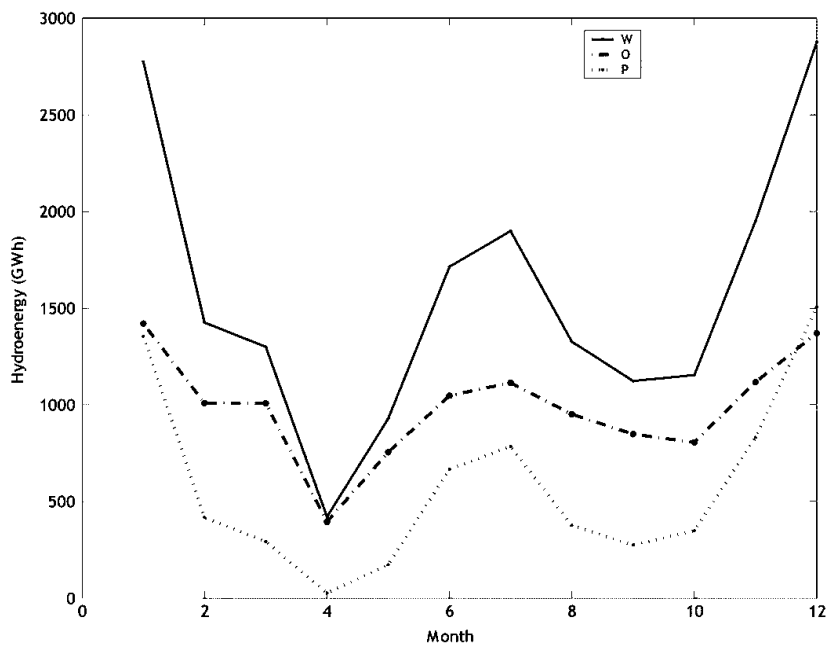

Fig. 5. Monthly optimal allocation of hydroenergy. Dry year.

can be used as starting point to establish maintenance schedules for the thermal units, system reliability analysis, as a tool for long-term price prediction and in expansion planning studies.

The analysis of a detailed example, using the Spanish generating system, the thermal and hydraulic characteristics of 2002, and the predicted demand for 2007, and a monthly time frame, illustrates the simplicity and application of the method. The results reveal the effects that water management policies have on the reliability index, and that the necessity of incorporating new thermal units in order to meet demand in 2007, especially for a dry year scenario.

\section{REFERENCES}

[1] M. Pereira, L. Barroso, and R. Kelman, "Market Power Issues in BidBased Hydrothermal Dispatch,", PSRI Tech. Rep., 2000.

[2] F. C. Schweppe, M. C. Caramanis, R. D. Tabors, and R. E. Bohn, Spot Pricing of Electricity. Norwell, MA: Kluwer, 1988.

[3] J. Juan and I. Ortega, "Reliability analysis for hydro-thermal systems including the effect of maintenance scheduling," IEEE Trans. Power Syst., vol. 12 , no. 4 , pp. 1561-1568, Nov. 1997.

[4] C. González and J. Juan, "Leveling reliability in systems with large hydro resources," IEEE Trans. Power Syst., vol. 14, no. 1, pp. 23-29, Feb. 1999.

[5] R. Billinton and R. N. Allan, Reliability Evaluation of Power Systems. London, U.K.: Pitman, 1984.

[6] R. Billinton and P. G. Harrington, "Reliability evaluation in energy limited generating capacity studies," IEEE Trans. Power App. Syst., vol. PAS-6, pp. 2076-2085, Dec. 1978. 
[7] R. L. Sullivan, Power System Planning. New York: McGraw-Hill, 1976.

[8] R. N. Allan, A. M. Leite da Silva, A. A. Abu-Nasser, and R. C. Burchett, "Discrete convolution in power system reliability," IEEE Trans. Rel., vol. R-30, no. 5, pp. 452-456, Dec. 1981.

[9] A. M. Leite da Silva, F. A. F. Pazo Blanco, and J. Coelho, "Discrete convolution in generating capacity reliability evaluation-LOLE calculations and uncertainty aspects," IEEE Trans. Power Syst., vol. 3, no. 4, pp. 1616-1624, Nov. 1988.

Camino González was born in Spain in 1964. She received the degree in nuclear engineering in 1987 and the Ph.D. degree in 1993 from the Universidad Politécnica de Madrid (UPM), Spain.

She is Associate Professor in the Statistics Department, UPM. Her current fields of interest include applied statistics and reliability analysis.

Jesús Juan was born in Spain in 1959. He received the degree in industrial engineering in 1983 and the Ph.D. degree in 1987 from the Polytechnical University of Madrid, Spain.

He is Professor in the Statistics Department, Polytechnical University of Madrid. His current fields of interest include applied statistics and reliability analysis.
José Mira was born in Madrid, Spain, in 1960. He received the degree in nuclear engineering in 1986 and the Ph.D. degree in applied statistics in 1995, both from the Polytechnical University of Madrid.

$\mathrm{He}$ is Associate Professor of statistics at the Polytechnical University of Madrid. His current research fields of interest are Kriging models, statistical analysis of computer codes and reliability of electric power generating systems.

Francisco J. Prieto was born in Madrid, Spain, in 1958. He received the B.Sc. degree in industrial engineering from UPM and the Ph.D. degree in operations research from Stanford University, Stanford, CA.

$\mathrm{He}$ is a Professor in the Statistics and Econometrics Department at Universidad Carlos III de Madrid. His current fields of interest include nonlinear optimization, decomposition algorithms, and large-scale optimization.

María J. Sánchez was born in Spain in 1964. She received the degree in electrical engineering in 1989 and the Ph.D. degree in applied statistics in 1995 , both from the Polytechnical University of Madrid.

She is Associate Professor of statistics at the Polytechnical University of Madrid. Her current research fields of interest are outliers in time series, Kriging models, and reliability of electric power generating systems. 